\title{
TOUCHING GRAIN KERNELS SEPARATION BY GAP-FILLING
}

\author{
MATThiEu FAESSEL ${ }^{1}$ AND FRANCIS COURTOIS ${ }^{2}$
}

${ }^{1}$ MINES Paristech, Centre de Morphologie Mathématique, Mathématiques et Systèmes, 35 rue Saint Honoré, 77305 Fontainebleau Cedex, France, ${ }^{2}$ UMR 1145, INRA, AgroParisTech, 1 Avenue des Olympiades, 91744 Massy Cedex, France e-mail: matthieu.faessel@ensmp.fr, francis.courtois@agroparistech.fr (Accepted September 29, 2009)

\begin{abstract}
Separation of touching grain kernels is a recurring problem in image analysis. Morphological methods to separate merged objects in binary images are generally based on the watershed transform applied to the inverse of the distance function. This method is efficient with roughly circular objects, but cannot separate objects beyond a certain elliptic shape nor when the contact zones are too numerous or too large. This paper presents a gap-filling method applied to the skeleton of the image background as an alternative technique to go further in the fused objects separation process. Open lines resulting from skeletonization are prolonged according to their direction from corresponding end points. If the distance between two lines is smaller than a certain value, their respective end points are connected. Results of combined use of watershed and gap-filling based methods are presented on sample binary images. An example of its use on an particularly complex image containing rice grains shows that it allows to segment up to $90 \%$ of the grains when classical watershed methods allow only to segment $25 \%$ of the grains. An application to breakage and cracks assessing of parboiled rice kernels is presented.
\end{abstract}

Keywords: gap-filling, mathematical morphology, overlapping grains, segmentation, skeleton.

\section{INTRODUCTION}

Fused grain kernels separation is a classical problem in binary images segmentation. For the general case, solutions are proposed with different approches (Schmitt and Hasse, 2009): contour or active contour based techniques, graph or level set approaches, parametric fitting or morphological methods. Morphological methods used to separate fused objects in binary images are generally based on the watershed transform (Beucher, 1990) on the complementary of the distance function (Talbot and Appleton, 2002). However, when fused particles have elliptic shape or when they are fused beyond a certain point, watershed algorithms may be insufficient to produce an appropriate segmentation (Shatadal et al., 1995; Talbot and Appleton, 2002; Wang and Paliwal, 2006). Fig. 1 shows the watershed transform on couples of circular fused objects with decreasing distances between their centers. As we can see, classical watershed based algorithms are effective even for an important degree of overlap $(d=1.2 R)$. Indeed, we know that as long as the centers of two disks lie on each side of their disecting line, watershed algorithm will succeed in separating them (Beucher and Vincent, 1990).

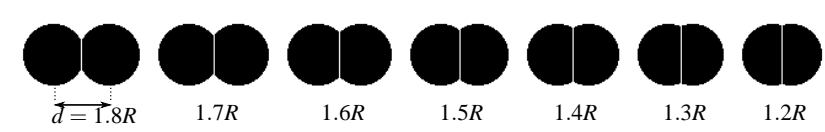

Fig. 1. Watershed based segmentation on circular fused objects.

On the contrary, Fig. 2 shows the result of watershed based segmentation on elliptical fused objects with a constant distance between their centers and a growing $R_{\max } / R_{\min }$ ratio.

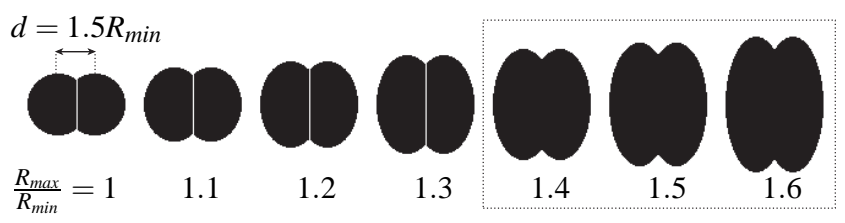

Fig. 2. Watershed based segmentation on elliptical fused objects.

It appears that above a certain shape factor, watershed algorithm fails to separate two overlapping objects.

Those functions operate on markers (intrinsic markers such as the maxima of the euclidean distance function, or markers defined arbitrarly by means of more elaboarted technics) of fused objects and their success depends on the ability to obtain as many markers as the number of fused kernels. Fig. 3 represents the ultimate points (the maxima of the distance function) of the elliptical fused kernels. 


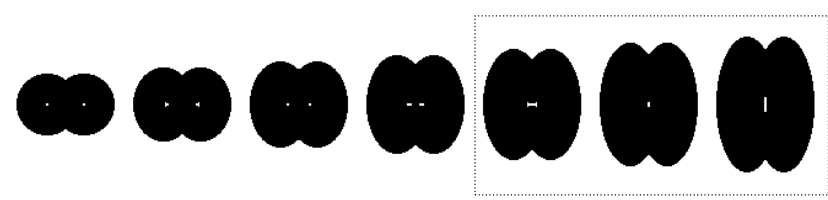

Fig. 3. Ultimate points (white) of elliptical fused objects (black).

It appears that the more the objects have an important $R_{\max } / R_{\min }$ ratio, the more their markers position converge until they merge. From that point, it is impossible for watershed based algorithm to detect more than a single kernel.

Several techniques have been explored to improve the determination of markers of elliptical objects: the use of the elliptical distance in place of Euclidean distance (Talbot and Appleton, 2002), the bisector (Meyer, 1979) or conditional bisector (Talbot and Vincent, 1992) functions, etc..., but those methods are inefficient or difficult to use when fused objects are numerous, not perfect disks, or when contact zones are important. It is also possible to use methods based on ellipse fitting (Talbot and Appleton, 2002; Evans et al., 2004; Zhang et al., 2005), which appear efficient as long as clusters contain only a few grains, or whose performance depends on the fit of the contour to an ideal ellipse (Baia et al., 2009).

When concavities corresponding to contact zones are important, the most evident solution remains to find a way to locate them and to link them together (Visen et al., 2001; van den Berg et al., 2002; Schmitt and Hasse, 2007). In this paper, we present a method using the skeleton of the background image to locate the concavities and to draw clipping lines, in order to improve the segmentation of non-circular fused objects in binary images.

The initial scope of this work was to develop image analysis techniques to quantify some quality parameters on rice grains: global properties (amount of broken kernels, ...), but also individual grain properties (dimensions, degree of crack-free kernels, ...), which implies to be able to individualize each grain of a sample.

\section{MATERIALS AND METHODS}

The material used in this study is based on long white rice grains of type "Ariete" (origin Camargue, France). The characteristic dimensions for this type of grains are $2 \mathrm{~mm}$ for the width and $7 \mathrm{~mm}$ for the length (which represents a $R_{\max } / R_{\min }$ ratio of 3.5).

\footnotetext{
${ }^{1}$ http://rsbweb.nih.gov/ij/index.html
}

The main purpose of this work was to develop a light and fast solution to extract individual properties such as length, width or state (broken, cracked,...) from a rice grain sample. Some studies give an important initial effort to position manually grains to make easier the stage of image analysis (Shatadal et al., 1995; van Dalen, 2004). In our case, the process had to be as simple and as fast as extract a grain sample from a batch, place it on the acquision system and launch the acquisition and analysis process. Furthermore, samples sometimes contain hundreds of grains and manual grain placement would lead to a non negligible waste of time and a need of an important surface for image acquisition.

The acquisition system is a simple standard flatbed scanner. Grains are tabled directly on the glass of the scanner and are quickly apportioned to avoid excessive overlapping. Then a black box is used to recover the grains and isolate them from ambient light. To reduce the size of the images, only a $115 \mathrm{~mm} \times 70 \mathrm{~mm}$ surface was used to place the grain sample (about 2 $3 \mathrm{~g}$ corresponding to about 200 grains) and a $300 \mathrm{dpi}$ resolution and 8 bits depth (grayscale) were used.

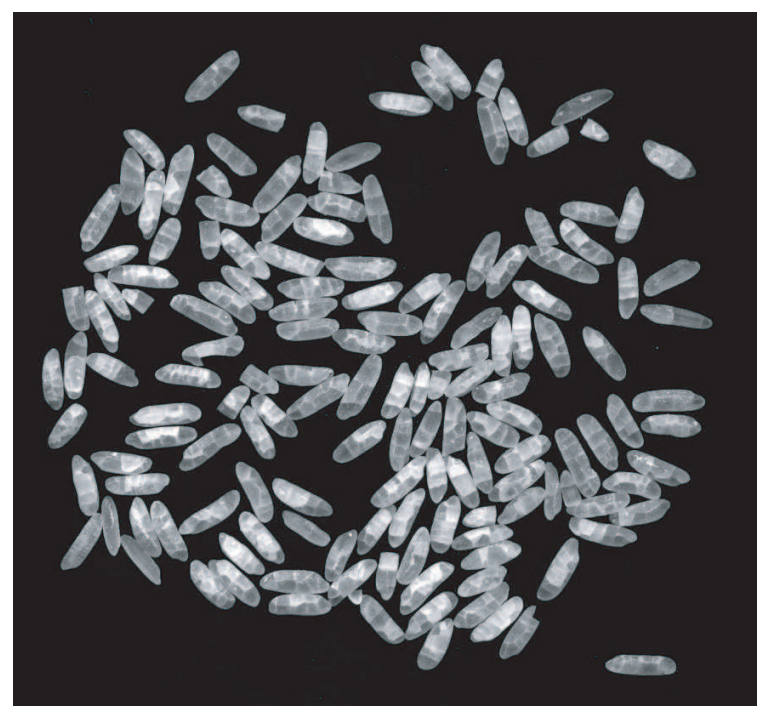

Fig. 4. Sample image obtained with the flatbed scanner $(850 \times 800$ pixels $)$.

The algorithms were developped and implemented under Java using the ImageJ software ${ }^{1}$ (Girish and Vijayalakshmi, 2004). ImageJ is a public domain Java image processing program inspired by NIH Image for the Macintosh and developped by Wayne Rasband at the Research Services Branch, National Institute of Mental Health, Bethesda, Maryland, USA. 


\section{IMAGE BINARIZATION}

It is important to notice that the information contained in the grey-level image is generally useful in order to split fused objects. In Fig. 5 is represented the morphological gradient calculated on the greylevel image. As we can see, gradient between grains is very weak compared to the variations corresponding to internal grain fissures and therefore can not be exploited to split grains.

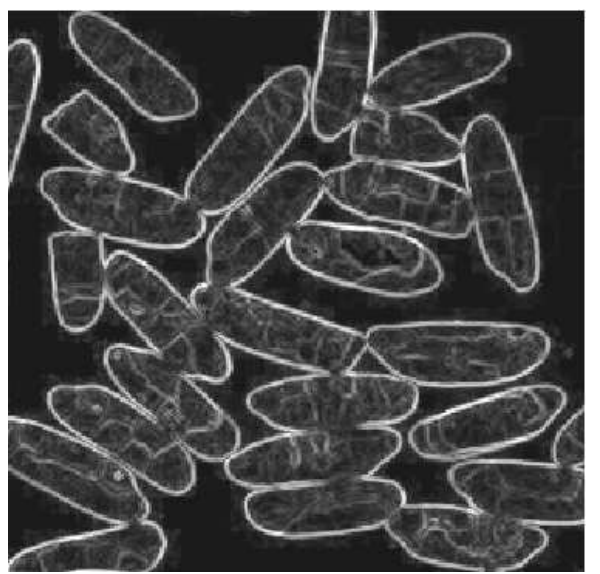

Fig. 5. Morphological gradient calculated on the greylevel image of Fig. 4 (detail).

As our images have two distinct classes of pixels (grains and background), we use the Otsu algorithm (Otsu, 1979), which allows to find automatically an optimum threshold. Ostu algorithm consists in separating the two classes so that their combined spread (within-class variance) is maximal. The resulting binary image is presented in Fig. 6 .

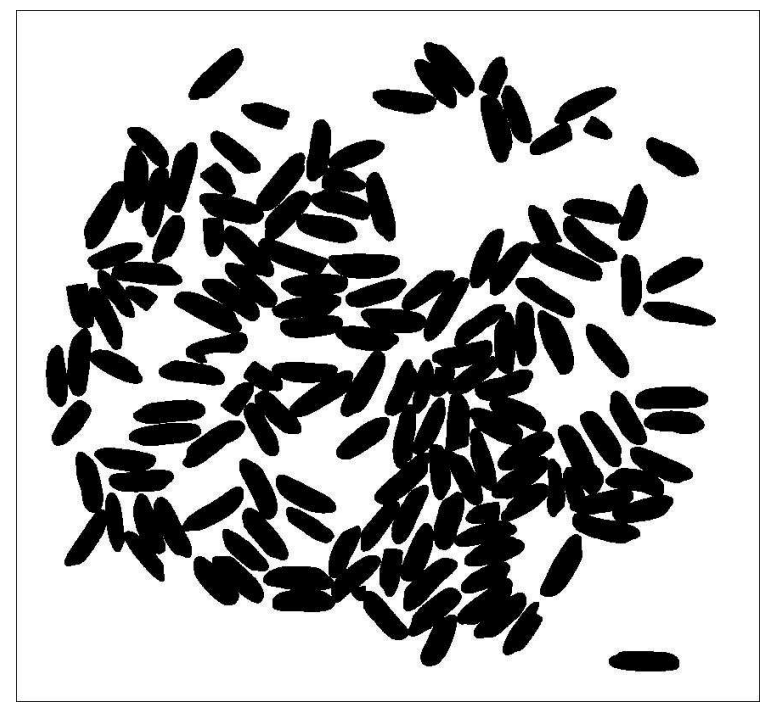

Fig. 6. Binary image obtained with the Otsu algorithm applied on the grey-level image (Fig. 4).

\section{CONCAVITIES LOCATION}

In order to locate concavities between grains, it is possible to use the cluster convex hull. Another possibility is to analyse the concavity of the contours of the grain clusters (Baia et al., 2009), but because of noise or of the image grid along contours, it is usual to detect false concave regions, and to obtain an overestimation of the number of concave points.

In our case, we use the morphological skeleton to detect the corners of the complementary image and then, extract the concave points from the skeleton open lines. The use of the morphological skeleton to detect object corners in a binary image can be found with different implementations (Dinesh and Guru, 2006). The main advantages are that it is a robust and nonparametric method.

The skeleton is calculated on the background of the binary image. It is obtained by means of a morphological thinning (Serra, 1982). The algorithm used here is the single pass thinning algorithm developped by Zhou et al. (1995). The result is presented in Fig. 7.

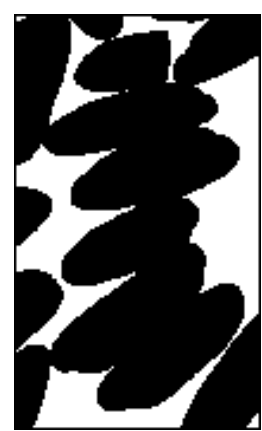

a

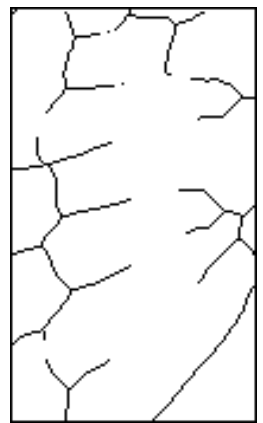

b

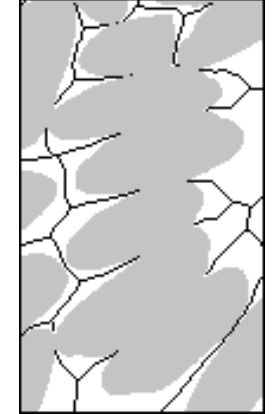

Fig. 7. Skeleton of the background image. (a) Binary image, (b) Background image skeleton (c) a over $b$.

\section{GAP-FILLING METHOD}

Automatic reconnection of linear segments is also a classical problem in image analysis. When portions of missing lines are sufficiently small, morphological operations such as closing (dilation followed by erosion) or localisation of nearest neighbours are commonly used in order to connect them (Zhang et al., 1999; Visen et al., 2001). In our case, the distance between the endpoints of the lines we want to connect is often larger than the width of the grains, and the size of closing necessary to link them would lead to completely fill skeleton cells.

The pairing procedure used here to join endpoints is quite similar to the "linking edge-chains" method 
described by Ferrari et al. (2006), except that we use 2D spherocylinders in place of isocele trapezia to extend and join open-lines. First, segment endpoints are localized. An "average" direction of the segment is calculated on a given length from its endpoint. Open lines are then linearly extended from their endpoint on a specified distance until they join (or until another extending line reach their neighborhood in a given radius).

\section{ENDPOINTS DETERMINATION}

Endpoints are detected from the skeleton thanks to the morphological hit-or-miss operation (Serra, 1982). Considering a couple of two disjoint structuring elements $T=\left\{T^{\prime}, T^{\prime \prime}\right\}$, the hit-or-miss operation is defined upon image $X$ as :

$$
\begin{aligned}
\eta_{T}(X) & =\left\{z: T^{\prime \prime}(z) \subseteq X^{c} ; T^{\prime}(z) \subseteq X\right\} \\
& =\varepsilon_{T^{\prime}}(X) \cap \varepsilon_{T^{\prime \prime}}\left(X^{c}\right)
\end{aligned}
$$

where $\varepsilon_{T^{\prime}}(X)$ and $\varepsilon_{T^{\prime \prime}}\left(X^{c}\right)$ are respectively the erosion of $X$ with $T^{\prime}$ and the erosion of the complementary image $X^{c}$ with the structuring element $T^{\prime \prime}$.

In order to extract the extremity of open lines (Fig. 9a), we use the hit-or-miss operation with a "U" structuring element and its seven successive rotations (Fig. 8).
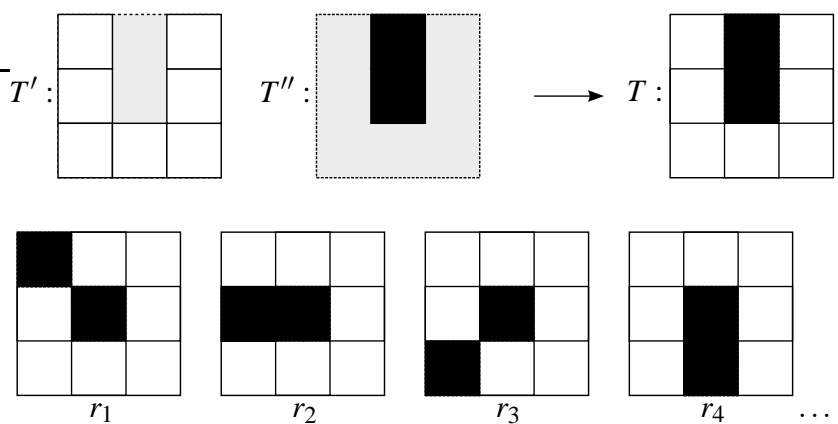

Fig. 8. Definition of the " $U$ " structuring element and its first rotations.

\section{LINES DIRECTION CALCULATION}

Starting from the endpoints, we apply successive morphological thinnings (Serra, 1982) with the same " $U$ " structuring element (and rotations) to thin the open lines on a certain length, or until idempotence (in this case, the junction of several lines). The thinning operation corresponds to the residue between an image and its hit-or-miss transformation :

$$
\Theta_{T}(X)=X \backslash \eta_{T}(X)=X \backslash\left[\varepsilon_{T^{\prime}}(X) \cap \varepsilon_{T^{\prime \prime}}\left(X^{c}\right)\right]
$$

The "average" direction of a line is calculated between an end point and the last point obtained by thinning the corresponding line (Fig. 9b).

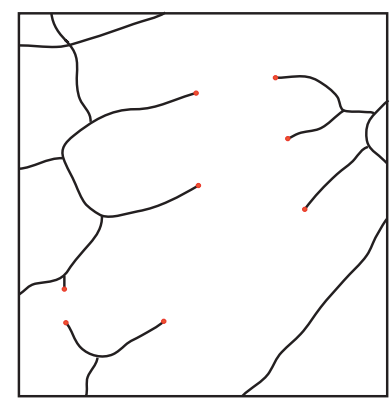

$\mathrm{a}$

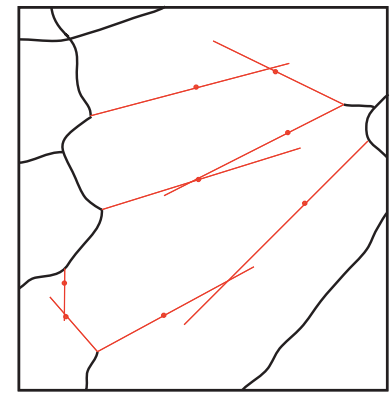

C

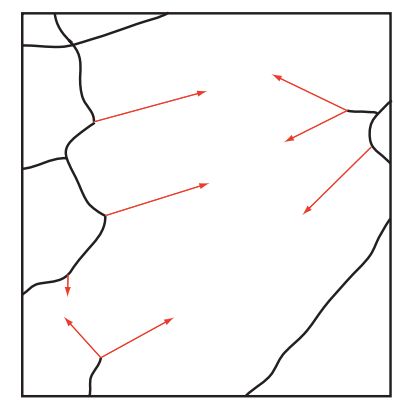

b

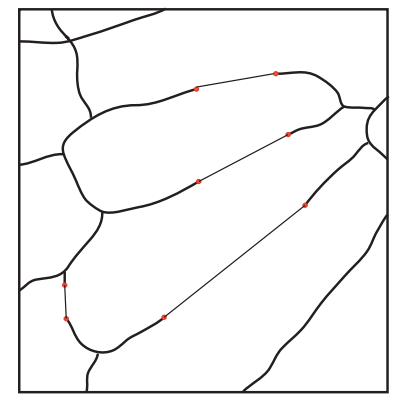

d
Fig. 9. Gap-Filling steps. a) End-points localisation. b) Open-lines direction evaluation. c) Open-lines prolongation. d) End-points junction.

\section{LINES PROLONGATION}

This line of average direction is then extended from terminal points on a fixed length. At every step of reconstruction, we extend the line of a point (pixel) and determine if it intersects with another one (Fig. 9c).

In order to detect the intersection between different lines, each line is represented on a black image with a different value. Each time we extend a line $i$ from one pixel, we check if the corresponding pixel on the black image has the value 0 or the value of another line $j$. In the first case, we continue the extending process, and in the second one, we define a new contact pair between lines $i$ and $j$. When a contact pair has been established, we draw as straight line between the corresponding endpoints. This line is then subtracted to the binary image to split the connex grains.

Note that line crossings are banned: when prolongating a line, if the next pixel corresponds to the skeleton or to a previous link, the prologation process of the line is stopped.

\section{IMPLEMENTATION}

During the pairing procedure, some problems can occur:

- two corresponding lines may never connect ; this happens if two lines are parallel or if they don't intersect between the two end-points. To ensure that two corresponding contact zone lines join, at 
each step of prolongation, we look for the presence of a nearby line within a radius of fixed size $R_{n}$ (Fig. 10).

As previously, each time we extend a line $i$ from one pixel, we check on the black image (on which extending lines are represented) the presence of a pixel with a different value in a radius of $R_{n}$. If a pixel with a value $j$ is found, we set a new contact pair between lines $i$ and $j$.

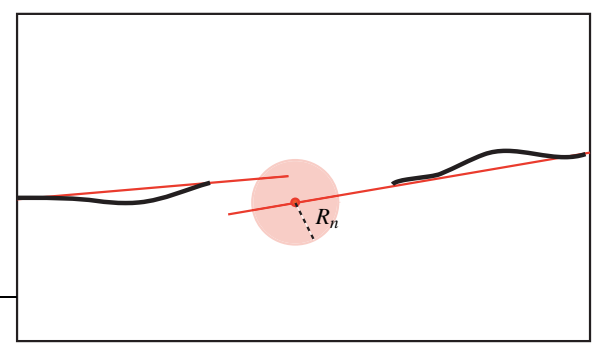

Fig. 10. Detection of two lines proximity.

- the average direction calculated from the endpoint to the open line root doesn't fit the global curvature of the line, which will be prolonged in an unexpected direction. For this reason, it is often better to calculate the average direction on a fixed length $L_{c}$ rather than on the whole length of the line (Fig. 11a).

- if a line is prolonged on a too long distance, unexpected contacts can occur between lines corresponding to different contact zones. To avoid this situation, lines are prolonged only on a fixed distance $L_{p}$, generally smaller than the specific length of a grain (Fig. 11b).

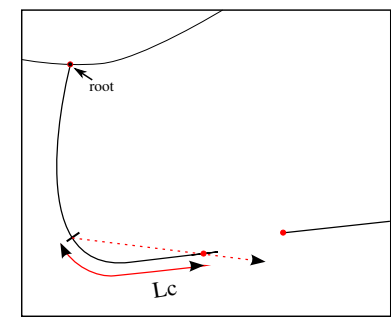

a

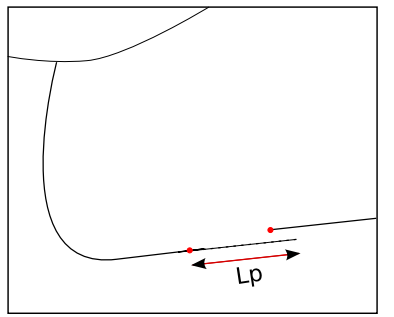

b
Fig. 11. Lenght of direction calculation a). Prolongation length $b$ ).

In order to obtain good results, it is necessary to execute the procedure sequentially with growing values for the parameters $L_{c}, L_{p}$ and $R_{n}$. First, small values of $L_{c}, L_{p}$ and $R_{n}$ are used in order to fill small gaps. Then the operation is repeated with higher values, to join progressively more distant lines. The maximum values for the parameters are respectively the specific length and width of a grain for $L_{p}$ and $R_{n}$ ( $L_{c}$ is limited by the length of the open-line).

The figure 12 illustrates the sequence of pairing procedures with growing parameters: at the first step, we use small values of $L_{p}, R_{n}$ and $L_{c}$. Only two lines (on the bottom left) are linked. At the second step, values chosen for the parameters allow to build the two upper links of the figure, and the last link is determined only at the third step. At each step, the particles revealed by the new links are measured. If their width and length are lower or equal to those of a typical grain, they are considered as correctly segmented and are removed from the image. The procedure is then continued with higher parameters, until the image is empty (or until the maximum values of parameters are reached).

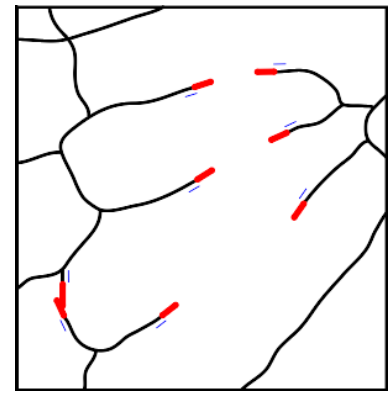

a

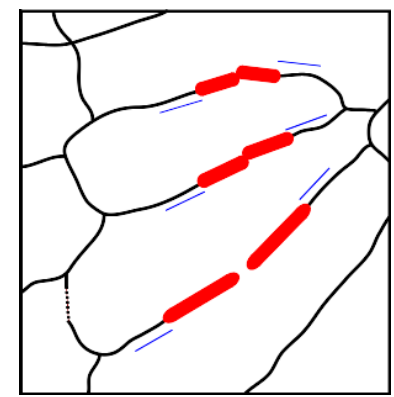

b

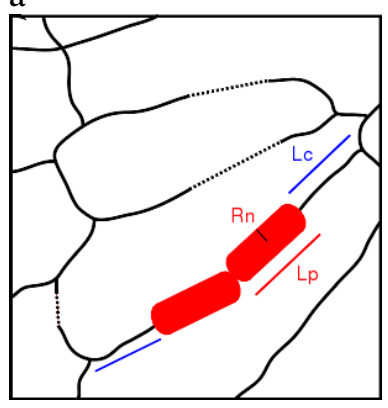

C

Fig. 12. Sequence of pairing procedures: a) First step with low values of parameters $L_{p}, R_{n}$ and $L_{c} . b$ ) Second step with medium values. c) Third step with high values.

The result of such a sequence applied on the image $7 \mathrm{a}$ is observed in Fig. 13. As we can see, the result is exactly what we expect to, except for the two grains on the top of the image which are not separated. In this particular configuration, the skeleton did not reveal two, but only one concavity (see Fig. 13a) and thus, only one open-line. Then, the left open-line can not be paired and no split line can appear. 


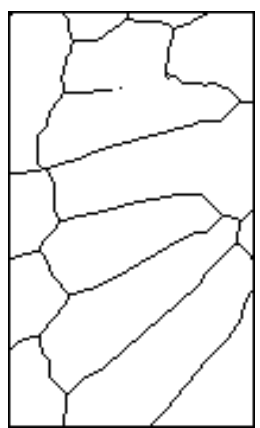

(a) - Gap-Filling.

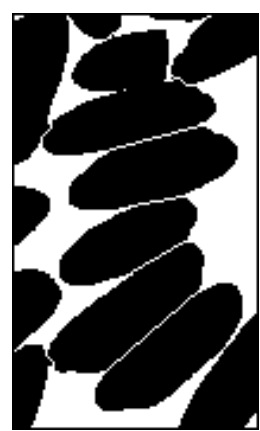

(b) - Result on binary image.
Fig. 13. Result of the gap-filling (a) and resulting grain splitting (b). Non splitted grains (at the top).

\section{APPLICATION TO BREAKAGE AND CRACKS ASSESSING OF PARBOILED RICE KERNELS}

The criterion used by industrials for evaluating quality of paddy rice is the measurement of the head rice yield (HRY, in \%) after milling. The rice is processed in an pilot rice-mill, generally consisting of a husker with rubber disks and a mill with an abrasive cone. Husked kernels are then separated in two fractions using a grain sorter: a whole fraction and a broken one. A kernel is considered broken if its length is smaller than $3 / 4$ of this of a whole kernel. The result is expressed in terms of rice processing yield, which is the ratio of the weight of white (husked) kernels to the total weight and in terms of head rice yield, corresponding also to the ratio of the weight of whole kernels to the total weight of white rice (norm ISO 6646:2000. Rice - Determination of the potential milling yield from paddy and from husked rice).

Our algorithm uses the same classification criteria: once the grains are individualized, they are classified according to their dimensions. The length of the grains is measured using the Feret's diameter. A grain is considered as broken if its length is smaller than $3 / 4$ of this of a whole kernel.

Furthermore, in order to characterize the cracking level of a grain, we evaluate the number of parts it contains. As we can see in Fig. 4, when a grain contains fissures, cracking zones present important grey level contrasts (due to different cracking planes orientation). Thus, parts can be easily identified using information such as the gradient. In order to close the contours corresponding to the different cracking zones of a grain, the gradient image is flooded by a watershed. To control the level of segmentation, the number of local minima is reduced using a gaussian filter on the gradient image before the flooding process. Once the different zones are revealed, the grains are classified according to the number of zones they contain: strongly cracked (more than 3 zones), slighly cracked (between 2 and 3 zones) and intact (1 zone).
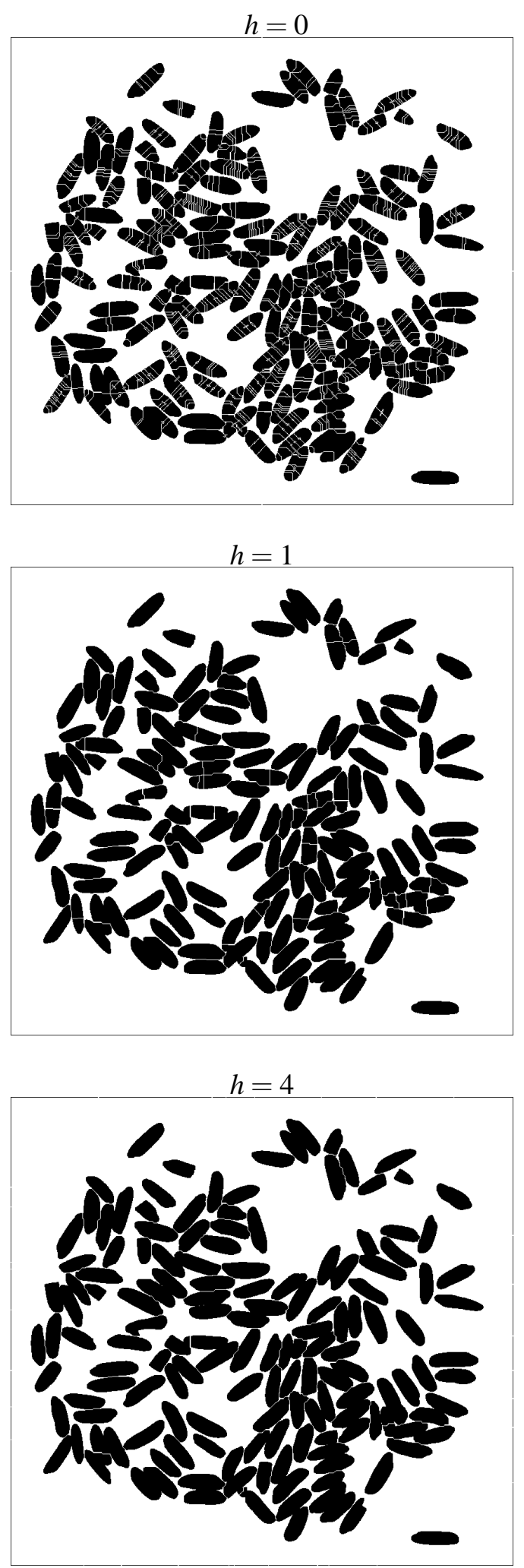

Fig. 14. Result of the segmentation obtained using the watershed transform on the distance function with markers corresponding to the h-minima of the distance function. 


\section{RESULTS}

In Fig. 14, we can see the segmentation obtained using the classical watershed transformation on the distance function on a grain sample image with a particularly difficult configuration: lots of conglomerates, important contact zones (only $15 \%$ of the grains are single). In order to control the level of segmentation obtained by the watershed, the markers used to perform the flooding are determined using the h-minima (Soille, 1999) of the distance function. As we can see, even with a relatively high value of $h(h=$ $4)$, there are still unwanted grain clippings. In order to completely avoid any inappropriate cuttings, we have to process the watershed with $h \geq 5$. This handles only very simple contact cases (clusters containing two or threee grains with small contact zones), and the amount of merged grains remains very large.

Fig. 15 represents the result of our method on the same image (resulting countours have been projected on the original grey-level image). Grains considered as successfully segmented are represented with a colored contour (in this example, grains are classified with a color code according to their state: blue for intact grains, green for broken, yellow and red for slightly and strongly cracked grains).

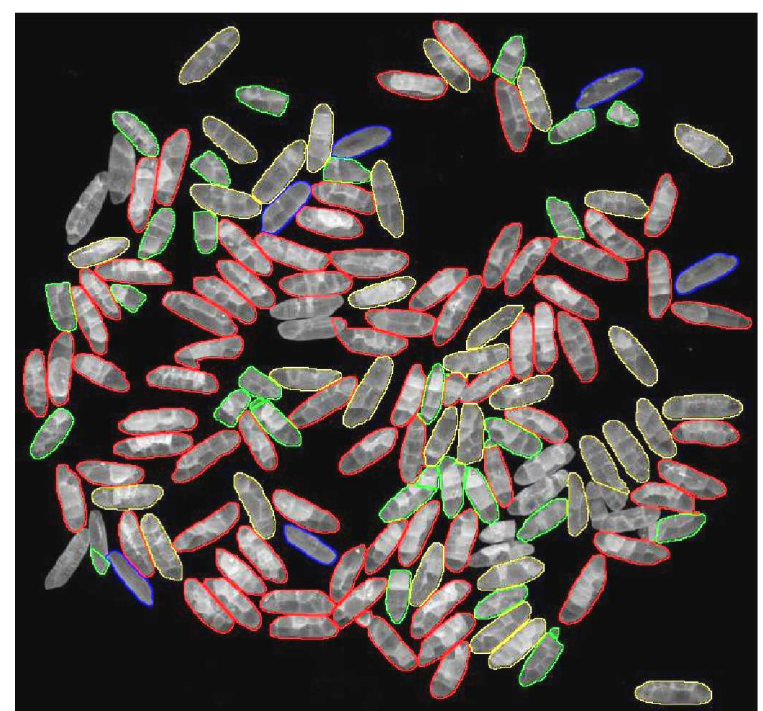

Fig. 15. Gap-filling based segmentation on a complex image of many touching rice grains. Grains considered as successfully segmented are analysed and represented with a colored contour: blue for intact grains, green for broken, yellow and red for slightly and strongly cracked grains.

We observe that most of the time, contours of grains are perfectly detected. Only a few grains are not correctly segmented. As it was said previously, this happends generally when concavities are not clearly revealed by the skeleton (inexistant or too short openlines).

The rate of successfully segmented grains is estimated using the ratio of the surface of correctly segmented grains to the surface of all the grains (easily determined by counting the foreground pixels on the binary image). In this case, segmentation based on watershed algorithms detects successfully only $25 \%$ of the grains total surface, while gap-filling algorithm reaches $90 \%$ of correctly segmented grains surface. In the case of more simple configurations, our algorithm allows us to obtain from $95 \%$ to $100 \%$ of successful segmentation (Fig. 16).

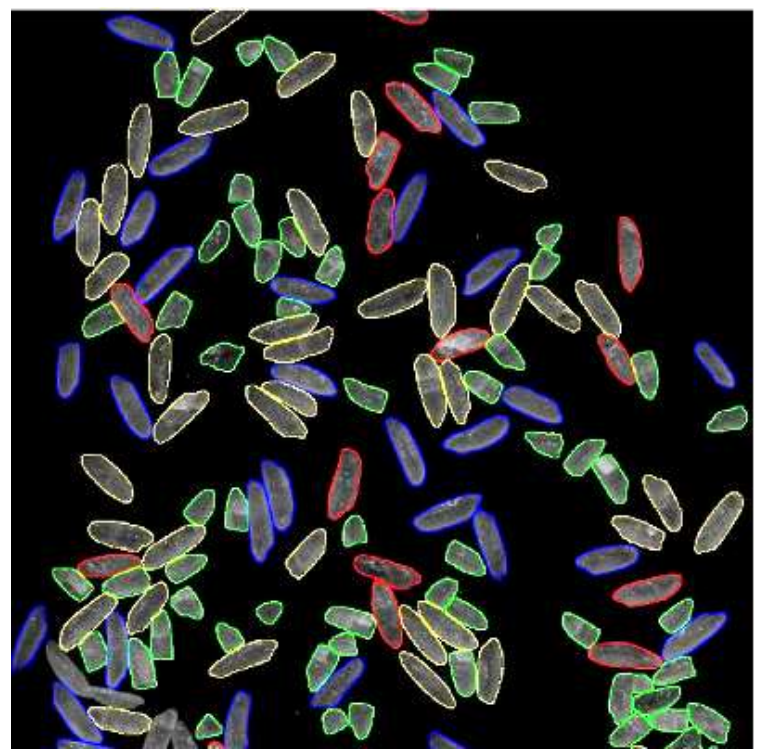

Fig. 16. Gap-filling based segmentation on a simpler case $(1000 \times 1000$ pixels $)$.

In order to validate the method, percentages of broken kernels obtained using image analysis were compared to measures obtained by manually separating and counting broken and intact grains. Different samples of processed, or not, parboiled rice dried in various conditions were used. As previously, each sample contains about 2-3 g (corresponding to about 200 grains). As we can see in Fig. 17, the estimates from the image analysis approach match perfectly both the measures using the ISO norm and the manual counting.

This algorithm was used on several hundred of images with more or less complicated cases, containing from ten to one thousand rice grains, with the same rate of success. The time needed to process one image depends of the number of grains and of the complexity of grains appointment, and is about several seconds (the time for the segmentation of the $850 \times 800$ pixels image in Fig. 15, is about 8 seconds on a standard laptop computer). 


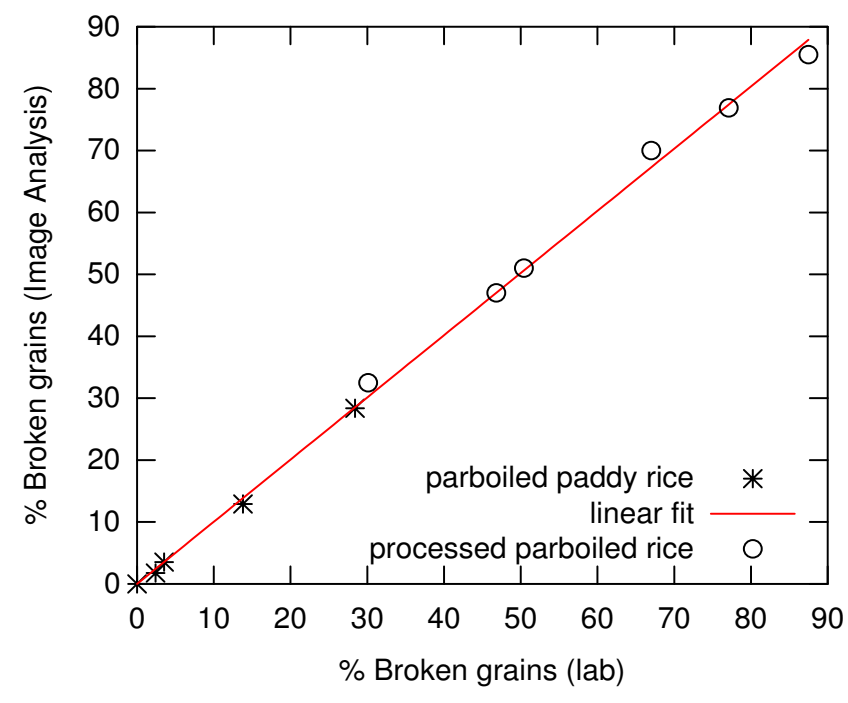

Fig. 17. Comparison between breakage ratios as obtained from laboratory visual inspection or ISO norm analysis, and sorting and image analysis estimation. The fitted slope of the line is $1.00434 \pm$ 0.00898 .

\section{CONCLUSION}

Separation of touching grains involves specific problems when grains have an elliptic shape and when clusters of grains contains many objects. We have seen that classical watershed based algorithms are not sufficient in this case. In this paper, a gapfilling method using the skeleton of the background image was introduced to draw clipping lines between touching grains. Then, a concrete implementation of the algorithm was presented in order to fulfil the separation process. Therefore, for images composed of many touching grain kernels with elliptic shapes, the described "gap-filling" based algorithm allows us to improve the segmentation process. More detailed application of this algorithm in food industry is presented in an upcoming article (Courtois et al., 2009).

\section{ACKNOWLEDGEMENTS}

The work was financially supported by the Office National Interprofessionel des Céréales (ONIC), France.

\section{REFERENCES}

Baia XZ, Sunb CM, Zhou FG (2009). Splitting touching cells based on concave points and ellipse fitting. Pattern Recogn 42:2434-46.
Beucher S (1990). Segmentation d'images et morphologie mathématique. Ph.D. thesis, Ecole des Mines, Paris, France.

Beucher S, Vincent L (1990). Introduction aux outils morphologiques de segmentation. In: ANRT, ed., Traitement d'image en microscopie à balayage et en microanalyse par sonde électronique. Paris.

Courtois F, Faessel M, Bonazzi C (2009). Assessing breakage and cracks of parboiled rice kernels by image analysis techniques. Food Control DOI: 10.1016/j.foodcont.2009.08.006.

Dinesh R, Guru D (2006). Corner detection using morphological skeleton: An efficient and nonparametric approach. In: Narayanan PJ, Nayar SK, Shum HY, eds., Comput Vis ACCV 2006. Lect Notes Comput Sci 3852:752-60.

Evans C, Berman M, Talbot H (2004). Offline fast object splitting. Tech. Rep. CMIS 04/55, CSIRO Mathematical and Information Sciences, North Ryde, NSW, Australia.

Ferrari V, Tuytelaars T, Van Gool L (2006). Object detection by contour segment networks. In: Leonardis A, Pinz A, eds., Computer Vision - Proc ECCV 2006. Lect Notes Comput Sci 3953:14-28.

Girish V, Vijayalakshmi A (2004). Affordable image analysis using NIH Image/ImageJ. Indian J Canc 41:47.

Meyer F (1979). Cytologie quantitative et morphologie mathématique. Ph.D. thesis, École des Mines de Paris.

Otsu N (1979). A threshold selection method from graylevel histograms. IEEE Trans Syst Man Cyb 9:62-6.

Schmitt O, Hasse M (2007). Radial symetries based decomposition of cell clusters in binary and gray level images. Pattern Recogn 41:1905-23.

Schmitt O, Hasse M (2009). Morphological multiscale decomposition of connected regions with emphasis on cell clusters. Comput Vis Image Und 113:188-201.

Serra J (1982). Image Analysis and Mathematical Morphology. London: Academic Press.

Shatadal P, Jayas D, Bulley N (1995). Digital image analysis for software separation and classification of touching grains: I. Disconnect Algorithm. Am Soc Agr Biol Eng 38:635-43.

Soille P (1999). Morphological Image Analysis: Principles and Applications. Springer-Verlag, 170-1.

Talbot H, Appleton BC (2002). Elliptical distance transforms and object splitting. In: Proc VI Int Symp Math Morph, April 3-5. Sydney, Australia.

Talbot H, Vincent L (1992). Euclidean skeletons and conditional bisectors. In: Vis Commun Image Proc '92. Proc SPIE 1818:862-76.

van Dalen G (2004). Determination of the size distribution and percentage of broken kernels of rice using flatbed 
scanning and image analysis. Food Res Int 37:51-8.

van den Berg E, Meesters A, Kenter J, Shlager W (2002). Automated separation of touching grains in digital images of thin sections. Comput Geosci 28:179-90.

Visen N, Shashidhar N, Paliwal J, Jayas D (2001). Identification and segmentation of occluding groups of grain kernels in a grain sample image. J Agr Eng Res 79:159-66.

Wang W, Paliwal J (2006). Separation and identification of touching kernels and dockage components in digital images. Can Biosyst Eng 48:7 (7 pp).
Zhang C, Murai S, Baltsavias E (1999). Road network detection by mathematical morphology. In: Proc ISPRS Worksh 3D Geospat Data Product Meeting Appl Requir, April 7-9. Paris, France.

Zhang G, Jayas DS, White NDG (2005). Separation of touching grain kernels in an image by ellipse fitting algorithm. Biosyst Eng 92:135-42.

Zhou RW, Quek C, Ng GS (1995). A novel single-pass thinning algorithm and an effective set of performance criteria. Pattern Recogn Lett 16:1267-75. 